\title{
Evaluation for Energy Saving Effect and Simulation Research on Vehicle Platoon Control for City Traffic
}

\author{
Takeki Ogitsu* \\ Gunma University, 1-5-1, Tenjin-cho, Kiryu-shi Gunma 376-8515, Japan \\ *Corresponding Author: ogitsu@gunma-u.ac.jp
}

\begin{abstract}
This paper reports on the development of vehicle behavior models for simulating vehicle platoon control to save energy on city roads and the effect on traffic flow. The demand for energy-saving technology for vehicles increases with each year. The automotive field has conventionally promoted energy efficiency by enhancing the physical performance of vehicles. In recent years, however, vehicle automation has made it easier to save energy by improving driving control. Such research and development includes vehicle platoon control. However, vehicle platoon control assumes use on highways because of the characteristics of the control. However, if vehicle platoon control is used in combination with eco-cruise control, it can also be used on city roads. In this study, simulations were performed to clarify the effect of combining eco-cruise control and vehicle platoon control on traffic flow. The background on energysaving technology for vehicles, especially vehicle control, is explained first. Related works on vehicle control to save energy on city roads are then explained, and their technological issues are clarified. Vehicle behavior models were designed to evaluate the effect of vehicle platoon control on city roads. Finally, a simulation was performed with vehicle platoon control using vehicle behavior models to evaluate the effect on traffic flow. The experimental evaluation confirmed the effect of vehicle platoon control on traffic flow city roads and clarified the relation between the target vehicle-to-vehicle (V2V) distance and traffic flow.
\end{abstract}

Keywords: Energy-saving technology, platooning, vehicle control, intelligent transport systems, intelligent driver model.

\section{Introduction}

The energy-saving performance of a technology is an important facet of its value. Automotive technology is no exception. The price of crude oil has greatly decreased in recent years. This is mainly because of the suppliers; for example, the production of shale oil has increased, and Saudi Arabia has not adjusted its production. The demand for oil by developed economies is decreasing, while the global demand is increasing. In addition, vehicle sales have been reported to be increasing due to the decrease in prices of crude oil in the USA ${ }^{(1)}$. This is why the automotive industry has an important role in the development of energy-saving technology.

Energy-saving technologies for vehicles have long been researched because they extend the driving distance, which is integral to a vehicle's value. The main energy-saving technologies for vehicles involve reducing the weight of the body, enhancing the engine efficiency, and decreasing the tire rotation resistance. Much research and development has been devoted to developing methods using these technologies, and they have contributed to improving the energy efficiency of vehicles. Recently, researchers have focused on saving energy through unconventional means, which has led to the development of technologies such as the idling stop mechanism and hybrid vehicles. In the intelligent transport systems (ITS) field, technologies are being developed to improve the energy efficiency of vehicles. ITS can save energy by using several kinds of information obtained from a traffic control center or onboard sensors to provide information for drivers or for driving control of the vehicle. 
This study considered using ITS to save energy. Vehicle platoon control is a control technology that allows a vehicle to follow another while maintaining a vehicle-to-vehicle

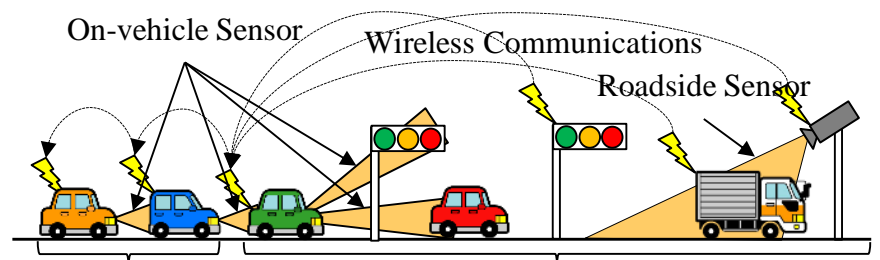

Platoon Control

(Saving Traffic Capacity)

(Considaration for Traffic Signals and Preceding Vehicles)

Fig. 1. Combination of eco-cruise control and vehicle platoon control.

(V2V) distance of several meters. If this control is used to follow a fast-moving vehicle, the following vehicle can utilize the slipstream of the preceding vehicle and save energy. This control can also reduce traffic congestion if used by several vehicles in a traffic flow because the vehicles can move in a higher density flow than with manual driving.

However, the main target of this control is highway use because the average velocity is lower and acceleration/deceleration is more frequent on city roads. The effect of the slipstream cannot be obtained when vehicles drive at lower velocities. In addition, the need for a vehicle to accelerate or decelerate greatly affects the energy-saving performance.

An eco-cruise control was developed to reduce unnecessary acceleration/deceleration on city roads. As illustrated in Fig. 1, when the leading vehicle of a traffic flow is driving with eco-cruise control and the following vehicles are driving with vehicle platoon control, the above weakness of the latter can be complemented by the advantages of the former. In this study, simulations were performed to evaluate the effect of combining vehicle platoon control with ecocruise control on a traffic flow.

Section 2 introduces related works on saving energy on city roads with vehicle control and explains technological issues. Section 3 specifies the vehicle behavior models combining eco-cruise control and vehicle platoon control for the simulations evaluating the effect on traffic flow. Section 4 reports on the experimental conditions and results of the simulation evaluation using the vehicle behavior models. Section 5 concludes this paper.

\section{Related Works}

This section presents related works on saving energy by vehicle control on city roads and clarifies the technological issues.

There has been much research and development on vehicle control technology to save energy in recent years. These studies can be classified into three kinds of controls: (a) considers static influences such as slopes and engine models, (b) considers foreseeable, dynamic influences such as traffic signals that are assumed to share information via communication, and (c) considers unforeseeable and dynamic influences such as general passenger vehicles and general traffic signals.

Kamal et al. ${ }^{(2)}$ proposed a control that considers static influences such as slopes and engine models. They validated their algorithm through a simulation.

Schwickart et al. ${ }^{(3)}$ realized a model predictive control that considers slopes and engine models. They used simulations based on the slope information of an actual rode to demonstrate that their proposed control algorithm can save energy.

Mahler and Vahidi ${ }^{(4)}$ proposed a predictive optimal velocity-planning algorithm that considers traffic signals which can obtain the traffic-signal phase and timing information. Thus, this traffic signal is a foreseeable and dynamic influence. They used a Monte Carlo simulation to validate their proposed algorithm.

Barth et al.$^{(5)}$ proposed a dynamic ecodriving velocity planning algorithm that considers traffic signals which can obtain traffic signal phase and timing information. The traffic signals are also foreseeable and dynamic influences. Their simulations showed that their algorithm improved the fuel economy and reduced $\mathrm{CO}_{2}$ emissions by around $12 \%$.

Huang et al. ${ }^{(6)}$ proposed an eco-friendly adaptive cruise control for electric cars that considers general vehicles, which are unforeseeable and dynamic influences. They performed simulations using MATLAB.

My research group developed eco-cruise control, which can save energy by considering both stable and dynamic influences, and performed simulations ${ }^{(7)}$.

Almost all research on vehicle control to save energy, including the above studies, only considered the energysaving effects of vehicles with the driving control installed. In contrast, we evaluated the energy-saving impact of our traffic control on the whole traffic flow ${ }^{(8)}$. Our results showed that, if the number of vehicles under eco-cruise control $^{(7)}$ in the traffic flow increases, this has a detrimental effect on the energy efficiency of the whole traffic flow. This is because each vehicle takes up more traffic capacity than 
usual driving by taking up greater $\mathrm{V} 2 \mathrm{~V}$ distances in order to save energy for the individual vehicle. Lower traffic capacity increases traffic congestion.

Combining eco-cruise control ${ }^{(7)}$ with platooning may increase the energy efficiency of the whole traffic flow. Research and development of platooning control, mainly for highways, has involved several approaches, such as the control design, impact on traffic flow, and acceptability to drivers $^{(9-12)}$. In addition, large research projects such as CHAUFFEUR, SARTRE, KONVOI, PATH, and Energy ITS have been promoted in different countries in recent years. Research has also gone into considering the stability and transfer sequence of platooning control ${ }^{(12,13)}$.

As explained above, platooning can save energy only when combined with eco-cruise control. However, no related study has clarified the relation between the platooning performance and effect on traffic flow when eco-cruise control and platooning control are combined. In this study, therefore, vehicle behavior models were developed that combine eco-cruise control and platooning control, and simulations were performed to evaluate the performance of the control and effects on the traffic flow.

\section{Vehicle Behavior Models}

In this section, details on the vehicle behavior models combining eco-cruise control and platooning control are explained. The below models were formulated for simulation, not for actual vehicle control. The eco-cruise control for actual vehicles would also need feedback control, which was neglected in the below models.

The eco-cruise control in this study was designed based on the intelligent driver model (IDM) ${ }^{(15)}$, which is a simulation model for the behavior of a general driver and considers preceding vehicles and traffic signals. IDM is an appropriate subject for the comparative evaluation presented in Section 4.

The eco-cruise control based on IDM comprised four behavior models: free road, considering unforeseeable and dynamical influences, considering foreseeable and dynamical influences, and platoon control. Each behavior model is detailed below.

\subsection{Free Road Behavior Model}

The free road behavior model simulates driving when there are no traffic signals and no other vehicles on the road. For the model developed in this study, the parameters were adjusted in order to more closely simulate driving behavior that minimizes the vehicle's fuel consumption when there are no traffic signals or other vehicles on the road.

\subsection{Behavior Model Considering Unforeseeable and Dynamical Influences}

The behavior model considering unforeseeable and dynamical influences simulates driving behavior that depends on the distance and velocity of unforeseeable influences in front of the vehicle. The design approach for this model is that the vehicle drives at a lower velocity than the velocity of all influences on the same line as the vehicle. With this approach, the $\mathrm{V} 2 \mathrm{~V}$ distance is equal to the margin for economical driving. For this behavior model, the parameters were adjusted to simulate driving behavior that minimizes fuel consumption by using economical methods such as fuel conservation and regenerative brakes.

\subsection{Behavior Model Considering Foreseeable and Dynamical Influences}

The behavior model considering foreseeable and dynamical influences simulates driving behavior where the vehicle passes greenlit traffic signals that offer information on the traffic-signal phase and timing to the vehicle. This model fixes the target velocity so that the vehicle arrives at the furthest greenlit traffic signal that can be reached with a single acceleration and low-speed driving. The acceleration depends on the characteristics of the free road behavior model and behavior model considering unforeseeable and dynamical influences.

\subsection{Platoon Control Behavior Model}

The platoon control behavior model simulates the driving behavior with platooning control when the nearest preceding vehicle is being driven under eco-cruise control or platoon control. When the nearest preceding vehicle is being driven under eco-cruise control or platoon control, the following vehicle behaves similarly to the nearest preceding vehicle. In other cases, the platoon control behavior model is excluded as a candidate vehicle behavior model.

In this study, the behavior model that induced the smallest acceleration was adopted for the vehicle's actual behavior. General vehicles not under the vehicle control proposed in this study were simulated with IDM.

\section{Simulation Evaluations}

A flat ring road that was $2 \mathrm{~km}$ in length was prepared to evaluate the effect of vehicle platoon control on traffic flow. 
A traffic signal was placed every $500 \mathrm{~m}$, and the traffic-signal phase and timing of each traffic signal were set to random numbers. The random numbers were normally distributed based on statistical information of the traffic signal timings for city roads with one lane in each direction in Tokyo, Japan. The random numbers were updated in each experiment. No vehicles flowed into/out of the road.

The driving behaviors of the simulated vehicles were classified into three patterns: (a) all of the vehicles drove based on IDM, (b) some vehicles drove based on IDM and the others were based on the vehicle behavior models excluding platoon control, and (c) some vehicles drove based on IDM and the others were based on the vehicle behavior models including platoon control. Pattern (c) was further divided into two sub-patterns: (c-1) the target V2V distance was set to $4 \mathrm{~m}$, and (c-2) the target $\mathrm{V} 2 \mathrm{~V}$ distance was set to $16 \mathrm{~m}$. The common model parameters for all of the simulation patterns were fixed to the same values. For patterns (b) and (c), some vehicles drove based on IDM, and the others drove based on the vehicle behavior models. The portion of vehicles driving based on the vehicle behavior models was varied from $50 \%$ by a uniform random number. For pattern (c), some vehicles drove based on IDM, and others drove based on vehicle behavior models including platoon control. The target $\mathrm{V} 2 \mathrm{~V}$ distance was selected from 4 and $16 \mathrm{~m}$ to evaluate the effect of traffic flow on vehicle platoon control. There were 50 vehicles on the road under each condition.

Each vehicle in the simulation was stopped at even intervals. After the simulation was started, the behavior model of each vehicle was updated at a calculation frequency of $1 \mathrm{~ms}$. In addition, each vehicle was simulated as having the fuel consumption efficiency of a D-segment vehicle.

The simulation ended when all of the vehicles drove 10 $\mathrm{km}$. Then, the average minimum required energy and average velocity were recorded. The results were averaged from 20 simulation runs.

\subsection{Simulation Results}

Fig. 2 shows the simulated average velocity and average minimum required energy for all traffic with the four patterns: (a), (b), (c-1), and (c-2).

The results confirmed that vehicle platooning increases traffic capacity through the combination of eco-cruise control and vehicle platoon control, which increases the energy-saving effect. This result suggests that more energy can be saved with a shorter V2V distance.

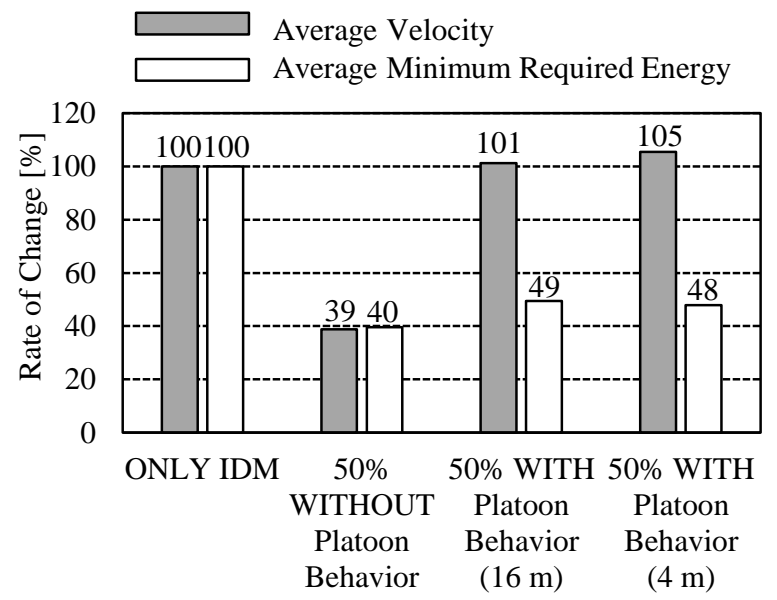

Fig. 2. Simulation results.

\section{Conclusions}

This paper reports the development of vehicle behavior models for simulating vehicle platoon control on city roads to determine the energy-saving effect.

The background on energy-saving technologies for vehicles are explained in the introduction. It is important for the leading vehicle to drive under eco-cruise control and for the following vehicles to drive under platoon control to save energy on city roads. Next, related works on vehicle control for saving energy on city roads are introduced, and the need for a quantitative evaluation of energy-saving methods on city roads is explained. Vehicle behavior models combining eco-cruise control and platoon control were designed to quantitatively evaluate the effect on traffic flow through simulation. Finally, a simulation evaluation using the vehicle behavior models designed in this study is presented. The results confirmed that the combination of eco-cruise control and platoon control has a significant effect on the traffic flow on city roads. The results suggest that the increased traffic capacity and energy-saving effect can be further enhanced by decreasing the target $\mathrm{V} 2 \mathrm{~V}$ distance of the vehicle platoon control.

Future work will involve validating the simulation evaluation in this study through the development of a vehicle control system based on the presented vehicle behavior models.

\section{References}

(1) Laura Clarke : "Oil Collapse Helps Make One Precious Metal a Little Rarer", Bloomberg Business, 


\section{http://www.bloomberg.com/news/, 2015}

(2) M.A.S. Kamal, M. Mukai, J. Murata, and T. Kawabe : "Ecological Vehicle Control on Roads With Up-Down Slopes", IEEE Transactions on Intelligent Transportation Systems, Vol. 12, No. 3, pp. 783-794, 2011

(3) T. Schwickart, H. Voos, J.R. Hadji-Minaglou, and M. Darouach : "An Efficient Nonlinear Model-predictive Eco-cruise Control for Electric Vehicles”, Proceedings of the 11th IEEE International Conference on Industrial Informatics, pp. 311-316, 2013

(4) G. Mahler and A. Vahidi : "An Optimal VelocityPlanning Scheme for Vehicle Energy Efficiency Through Probabilistic Prediction of Traffic-Signal Timing”, IEEE Transactions on Intelligent Transportation Systems, Vol. 15, No. 6, pp. 2516-2523, 2014

(5) M. Barth, S. Mandava, K. Boriboonsomsin, and X. Haitao : "Dynamic ECO-driving for Arterial Corridors", Proceedings of the IEEE Forum on Integrated and Sustainable Transportation Systems, pp. 182-188, 2011

(6) Jun Huang, Botao Zhu, Xiaohui Guo, and Zujue Chen : "The Concept and Simulation of Eco-friendly Adaptive Cruise Control", Proceedings of IEEE International Electric Vehicle Conference, pp. 1-5, 2012

(7) T. Ogitsu and M. Omae : "A Study of Energy-saving Effect of Longitudinal Control Based on Local Traffic States for Energy Saving," JSAE Transaction, Vol. 43, No. 2, pp. 561-566, 2012

(8) T. Ogitsu and H. Mizoguchi : Eco-Cruise Control by Combining Considerations of Traffic Conditions and Platooning, Proceedings of the 22nd ITS World Congress, Bordeaux, France, October, 2015

(9) J. Larson, K. Liang, and K. Johansson : “A Distributed Framework for Coordinated Heavy-duty Vehicle Platooning," Special Issue of IEEE Transactions on Intelligent Transportation Systems, CD-ROM

(10) S. Joo, X. Lu, and J. Hedrick : "Longitudinal Maneuver Design in Coordination Layer for Automated Highway System", Proceedings of the 2003 IEEE American Control Conference, pp. 42-47, 2003

(11)S. Tsugawa : "A History of Automated Highway Systems in Japan and Future Issues," Proceedings of the IEEE International Conference in Vehicular Electronics and Safety, pp. 2-3, 2008

(12) S. Tsugawa : "Results and Issues of an Automated Truck Platoon within the Energy ITS Project," Intelligent Vehicles Symposium Proceedings, pp. 642-647, 2014
(13) T. Ogitsu, M. Omae, and H. Shimizu : "State Estimation Algorithm for Compensating Failure of Position and Relative Position Sensors during Platooning using Intervehicle Communication", Transactions of the Society of Automotive Engineers of Japan, Vol. 39, No. 6, pp. 223228,2008

(14) T. Ogitsu, T. Hirano, and M. Omae : "Design and Evaluation of Transitional Process of Platooning of Heavy-Duty Vehicles", Proceedings of the 18th World Congress on Intelligent Transport Systems, CD-ROM, 2011

(15) Treiber Martin, Hennecke Ansgar, and Helbing Dirk, "Congested traffic states in empirical observations and microscopic simulations", Physical Review E, Vol. 62, No. 2, pp. 1805-1824, 2000 